\title{
Evaluation of tendinosis of the long head of the biceps tendon by strain and shear wave elastography
}

\author{
Mehmet Hamdi Şahan¹, Mikail İnal', Veysel Burulday¹, Turgut Kültür²
}

${ }^{1}$ Department of Radiology, ${ }^{2}$ Department of Physical therapy and rehabilitation, Kirikkale University School of Medicine, Kirikkale, Turkey

\begin{abstract}
Aim: To investigate strain (SE) and shear wave elastography (SWE) characteristics of the long head of the biceps tendon (LHBT) tendinosis in comparison with magnetic resonance imaging (MRI) findings. Material and methods: Twenty patients with a MRI diagnosis of tendinosis and twenty healthy subjects with normal LHBT in MRI were prospectively examined by SE and SWE. SE color mapping was divided into four types in accordance with elasticity designs: type I predominantly blue (hardest tissue), type II predominantly blue-green (hard tissue), type III predominantly green (intermediate tissue), type IV predominantly green-yellow-red (soft tissue). Quantitative measurements of LHBT hardness with SWE were analyzed in kilopascals $(\mathrm{kPa})$. Results: In the tendinosis group SE types in transverse scan were I in $24 \%$ of tendons, II in $50 \%$, III in $25 \%$, and in longitudinal scan I in 15\%, II in 75\%, and III in 10\%. In the control group SE types in transversescan were II in $10 \%$ of tendons, III in 55\%, IV in 35\%, and in longitudinal scan II in 10\%, III in 55\%, and IV in 35\%. SWE values in transverse scan were $38.32 \pm 7.2 \mathrm{kPa}$ in the tendinosis group and $18.6 \pm 3.1 \mathrm{kPa}$ in the control groupand in longitudinal scan $39.42 \pm 7.4 \mathrm{kPa}$ in the tendinosis group, and $20.62 \pm 4.6$ in the control group. There was a statistically significant difference in terms of elasticity patterns between the tendinosis and control groups $(\mathrm{p}<0.001)$. The receiver operating characteristic curve analysis was perfect and a cut-off value of tranverse $25.8 \mathrm{kPa}$ and longitudinal, $24.6 \mathrm{kPa}$ shear values had very high sensitivity and specificity for tendinosis. Conclusion: SE and SWE may be useful diagnostic tools for LHBT tendinosis when considering usability, cost effectiveness, and patient preference compared to MRI.
\end{abstract}

Keywords: biceps; tendinosis; magnetic resonance imaging; sonoelastography; shear wave

\section{Introduction}

Tendinopathy of the long head of the biceps tendon (LHBT) is an important source of pain in the anterior shoulder [1,2]. LHBT pathologies, mostly associated with rotator cuff tears and superior labrum anterior to posterior (SLAP) lesions, are also similar when clinically characterized $[1,3,4]$. Accurate diagnosis of shoulder tendon pathology for choosing medical or surgical treatment, as well as for specific planning of surgical proce-

Received 06.11.2017 Accepted 19.01.2018

Med Ultrason

2018, Vol. 20, No 2, 192-198

Corresponding author: Mehmet Hamdi Şahan, MD, assistant professor Kirikkale University School of Medicine, Department of Radiology 1450 Yahsihan / Kirikkale, Tukey

Phone:+90 505 6480687, +90 3183335011

E-mail: drmehmetsahan@hotmail.com dures is needed [5]. Conventional ultrasonography (US) and magnetic resonance imaging (MRI) are commonly used as noninvasive methods in patients diagnosed with tendinopathy of the LHBT.

MRI and US have been applied due to many advantages, including usability, cost effectiveness, and patient preference. However, tendinitis and tendinosis lesions are difficult, and in some cases impossible, to diagnose with conventional US since it usually shows the same echogenicity as the surrounding healthy tissue in the case of tendinopathy $[6,7]$. In addition, US is dependent on the practitioner. MRI is a noninvasive gold standard diagnostic modality that provides information on LHBT, rotator cuff tendons, soft tissues, and bone structures $[6,8]$.

Sonoelastography is a new, non-invasive US method used for evaluation of the elastic properties of tissues based on static compression and cross-correlation methods, qualitatively and/or quantitatively. The main elasto- 
graphic techniques include strain elastography (SE), in which the tissues are compressed axially, and shear wave elastography (SWE), which uses waves that are generated by transducers and interact with the tissue [9-13] Elastography has been successfully applied to lesions of the breast, prostate, pancreas, lymph nodes, thyroid gland, testes, and liver [9-13]. Nevertheless, it is not yet routinely used in clinical practice in the diagnosis of tendinopathy.

However, to our knowledge, there has been no study usingboth SE and SWE in LHBT tendinopathies. The purpose of ourstudy was to investigate the SE and SWE characteristics of LHBT tendinopathies in comparison with MRI.

\section{Material and methods}

\section{Study population and inclusion criteria}

We reviewed patients referred to our clinic between January 2017 and July 2017 with shoulder pain and tendinosis detected by MRI examination. Patients with anterior shoulder pain and dysfunction for 2-12 months were enrolled. A total of 158 shoulder MRIs of 158 patients were reviewed; among them 20 patients with LHBT tendinosis who complied with the criteria of our work and agreed to participate in the study and 20 control subjects with normal shoulder MRI were included in this study. A total of 40 shoulder previous examined by MRIs were enrolled. To reduce the effect of gender on the results, the number of male and female was equalized in both tendinosis and control group. LHBT tendinosis and the control group were prospectively examined by sonoelastography. In the patient and control groups, the dominant handwas included in the study of the LHBT. There are studies in the literature that show differences between dominant hand muscle strength and size [14-16]. Therefore, we preferred the LHBT on the side of the dominant hand. The study was approved by the hospital Ethics Committee and was conducted according to the Helsinki Declaration. Voluntary informed consent was obtained from all patients included in the study (Ethics committee decision no: 04/05, date 07.02.2017).

\section{Exclusion criteria}

Subluxation and dislocation in the LHBT were excluded since this would cause tension on the tendon. Acute trauma, LHBT tendon ruptures, adhesive capsulitis, ipsilateral passed shoulder surgery, pseudo-palsy with massive rotator cuff tear, advanced osteoarthritis, rotator cuff muscle atrophy, fatty degeneration with massive rotator cuff tear (greater than $2 \mathrm{~cm}$ ), muscle diseases, dermatomyositis and polymyositis were also excluded from the study.

\section{MRI examinations and interpretation}

MRI was performed with a 1.5 Tesla MR device (Philips MRI Systems, Achieva Release 3.2 Level 201310-21, Philips Medical Systems Nederland B.V.) using the surface shoulder coil. In the examination, T1 weighted: T1-TSE (Turbo Spin-Echo) axial, T1-TSE oblique coronal (780/15; FOV $14 \mathrm{~cm}$; cross-sectional thickness $3.5 \mathrm{~mm}$; intersection gap $0.4 \mathrm{~mm}$; matrix $320 \times 256$ ), T2 weighted: T2-FFE (Fast-Field Echo) axial, T2-TSE oblique sagittal, T2 weighted fat-suppressed, T2-SPAIR (spectral attenuated inversion recovery) axial and oblique coronal (3400/50; FOV, $14 \mathrm{~cm}$; cross-sectional thickness $3.5 \mathrm{~cm}$; intersection gap $0.4 \mathrm{~mm}$; matrix, $256 \times 256$ ) images were obtained. All MRIs were examined by common consensus by two radiologists experienced with the musculoskeletal pathology (M.H.Ş, M.I. 2-5 years experience in the musculoskeletal system). On MRI, if no tear of the LHBT tendon was detected, but fluid in the tendon sheath, increased signal and thickness of the tendon, this was intepreted as tendinosis (fig 1). Thickness was transverse measured at a distance of $1-2 \mathrm{~cm}$ distal to the insertionof the LHBT.

\section{Real-time sonoelastography and US examinations}

Sonoelastography examinations of all participants were carried out by one of two radiologists (M.H.Ş, V.B: 3 years experience in elastography with 10 years of conventional US experience) using a LOGIQ E9 sonographic system (GE Healthcare) with elastography software and a linear array probe of 9L, 6-15L MHz. All participants were in a sitting position, the arm in a neutral position, the forearm on the thigh, with the fingers in supination [17]. This examination was standardized according to a previously published technique [18]. The probe was placed anterior to the transverse and longitudinal shoulder. The

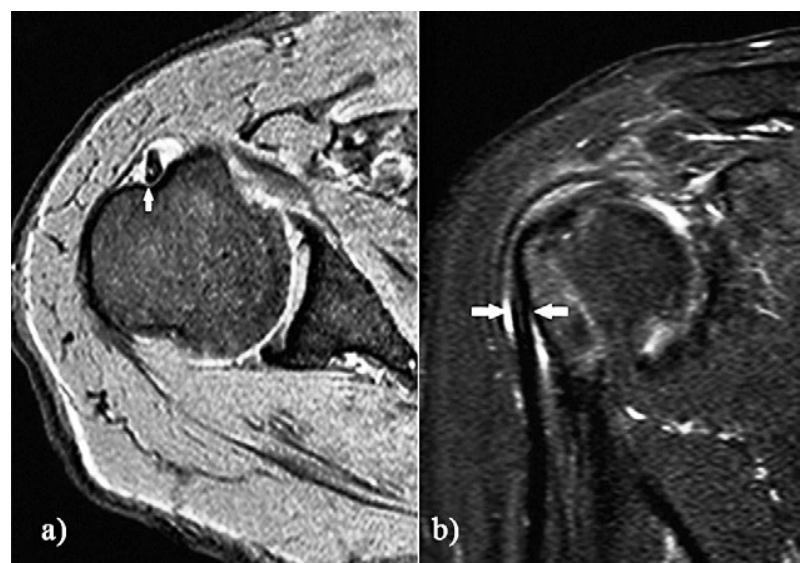

Fig 1. A 69 year-old male with LHBT tendinosis a) 2-FFE axial MRI image and b) T2-SPAIR coronal MRI image showing thickening, signal increase, and fluid around the tendon (white arrows). 


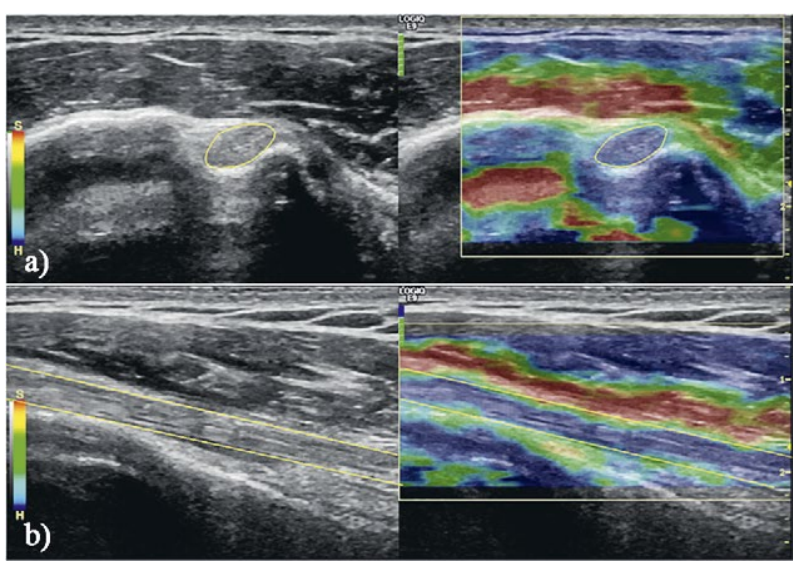

Fig 2. A 52 year-old female with LHBT tendinosis, strain elastography, a) transverse and b) longitudinal scan with type I (blue) elastographic patterns of the tendon.

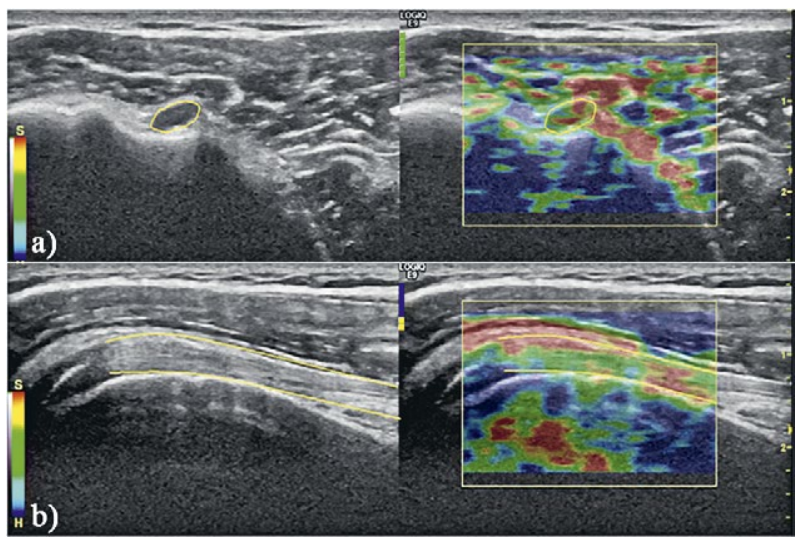

Fig 3. A 47 year-old male with normal LHBT, strain elastography: a) transverse scan, type IV patterns (green-yellow-red) of the tendon; b) longitudinal scan type III pattern (green-yellowred) of the tendon.

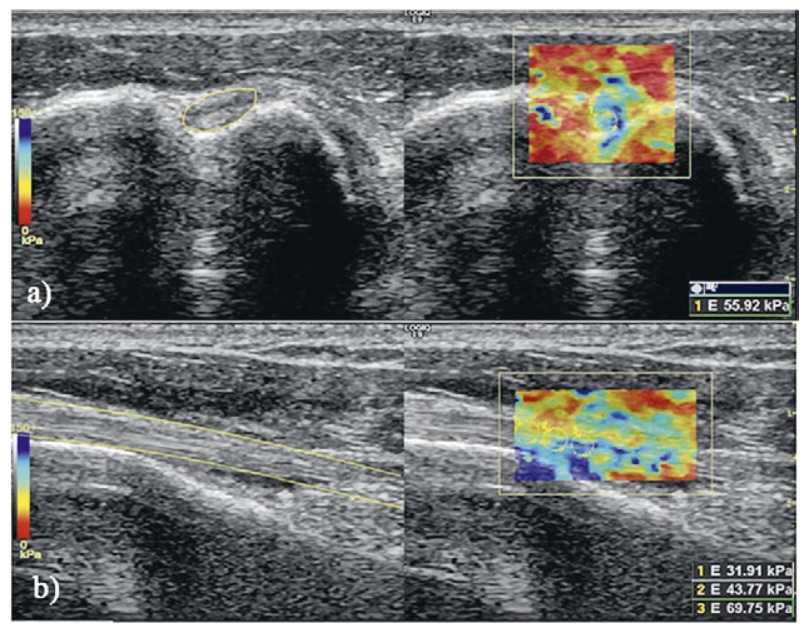

Fig 4. A 52 year-old female with tendinosis of the LHBT, SWE in a) transverse and b) longitudinal scan.
LHBT was examined in the bicipital groove, transverse and longitudinal plane. In US examination, tendon echogenicity, thickness and fluid in the tendon sheath were evaluated. Thickness was transverse measured at a distance of 1-2 cm distal to the insertion of the LHBT. Bmode US was performed followed by SE and SWE, in the same time. B-mode and elastographic images were shown simultaneously side by side on a split-screen display. Images in SE were performed by applying mild compression of the transducer with the hand-held device. The compression bar indicator of 1 to 7 was monitored in real time on the screen, and images were analyzed when the ideal compression was applied, which was in the 5-7 bar range. Elastography images according to the different levels of strain were displayed on the picture over a grayscale background in a color diagram that included red (softest component), green (intermediate stiffness) and blue (hardest component) [11]. SE patterns of the LHBT of all participants were interpreted by two radiologists with a common consensus. LHBT SE color mapping was divided into four types: type I predominantly blue (hardest tissue), (fig 2), type II predominantly blue-green (hard tissue), type III predominantly green (intermediate tissue), type IV predominantly green-yellow-red (soft tissue) (fig 3).

The images were saved in the transverse and longitudinal plane from inside the equator of the LHBT.

Quantitative analysis of LHBT hardness with SWE used kilopascal $(\mathrm{kPa})$ measurement with a color scale of 0-150 $\mathrm{kPa}$ with a circular region of interest (ROI) area of 2-4 $\mathrm{mm}$ diamenter, as displayed in figure 4 . In the longitudinal plane the measurements were performed at $1-4 \mathrm{~cm}$ distance from the proximal limit of the bicipital groove. In the transverse plane, for measurement the place where the tendon was most prominent in the bicipitalgroove was chosen. Sonoelastography properties were decided in consensus by two radiologists. The quantitative value for LHBT was obtained with at least three measurements and the mean values were used for the statistical analysis.

\section{Statistical analysis}

Statistical analyses were performed using the SPSS software package program (IBM), version 20.0. Data are expressed as the arithmetical mean \pm standard deviation (SD) or median (range), depending on normality as determined with the Shapiro-Wilk test. Differences between genders and the distribution of groups was compared using the Chi-square test. Elasticity designs were investigated using the chi-square test to assess the relationships between them, and we classified primary color designs and color designs to appraise the diagnostic ability of SE. The student's t-test was used to compare age, body mass 
index (BMI), LHBT thickness (in US and MRI), and SE and SWE of tendinosis of LHBT and control groups. A value of $p<0.05$ was considered statistically significant.

Receiver operating characteristic (ROC) curve analysis was performed to define the diagnostic significance of SWE values. ROC curves were used for the definition of the diagnostic sensitivity, specificity, positive predictive value (PPV) and negative predictive value (NPV). Subsequently, the cut-off value of SWE for differentiation between tendinosis of the LHBT and the control group was determined.

\section{Results}

The age, gender, BMI, dominant hand, and US and MRI tendon thickness in the LHBT tendinosis group and control group are summarized in table I.

MRI examinations showed tendon signal increase in 18 patients, irregular thickening of tendon in 2 patients, and fluid in all tendon sheaths in LHBT tendinosis group. Signals of all tendons were normal, and no fluid was seen in the tendon sheaths in the control group. There wasdiffuse dishomogeneity in 12 tendons, focal dishomogeneity increase in 5 tendons, fluid in the sheath of all tendons, while 3 tendon echogenicity were normal in the US examinations in the LHBT tendinosis group. Echogenicity of all tendons were normal, and no fluid was seen in the tendon sheaths in the control group.

SE characteristics in transverse and longitudinal images between the two groups are shown in table II. SWE characteristics in transverse and longitudinal images between the two groups are shown in table III.

ROC curve analysis gave a value of transverse images of $0.995(\mathrm{p}<0.001 ; 95 \% \mathrm{CI}=0.982-1.000)$ and longitudinal images of $0.981(\mathrm{p}<0.001 ; 95 \% \mathrm{CI}=0.949-1.000)$. For the tendinosis group the shear value of $25.8 \mathrm{kPa}$ in the transverse plan had a very high sensitivity and specificity (100\%), NPV, and PPV $-100 \%$ for all. The cut off value of $24.6 \mathrm{kPa}$ in the longitudinal plan had also a high sensitivity and specificity, NPV, and PPV: 100\%, 90\%, $100 \%$, and $90.91 \%$ respectively.

Table I. Comparison of age, gender, BMI, dominant hand, US and MRI tendon thickness between LHBT tendinosis and control groups.

\begin{tabular}{llll}
\hline Measurements & LHBT tendinosis $(\mathbf{n = 2 0})$ & Control group $(\mathbf{n = 2 0})$ & P values \\
\hline Gender (M:F) & $10: 10$ & $10: 10$ & 1.00 \\
Age (years) & $54.55 \pm 10.1$ & $47.55 \pm 11.9$ & 0.53 \\
BMI (kg/m $\left.{ }^{2}\right)$ & $27.15 \pm 4.12$ & $27.55 \pm 4.77$ & 0.77 \\
US tendon thickness (mm) & $2.88 \pm 0.42$ & $2.29 \pm 0.3$ & $<0.001$ \\
MRI tendon thickness (mm) & $2.8 \pm 0.54$ & $2.17 \pm 0.24$ & $<0.001$ \\
Dominant hand & 5 Left:15 Right & 3 Left:17 Right & 0.695 \\
\hline
\end{tabular}

The results are expressed as mean \pm standard deviation; LHBT - long head of the biceps tendon; M - Male; F - Female; BMI - Body mass index; US - Ultrasonography; MRI - Magnetic resonance imaging

Table II. Distribution of strain elastography patterns in LHBT tendinosis and control groups.

\begin{tabular}{|c|c|c|c|c|c|}
\hline \multirow[t]{2}{*}{ Paterns } & \multicolumn{2}{|c|}{ LHB tendinosis } & \multicolumn{2}{|c|}{ Control group } & \multirow[t]{2}{*}{ P values } \\
\hline & Transverse & Longitudinal & Transverse & Longitudinal & \\
\hline Blue (type I) & $5(25)$ & $3(15)$ & $0(0)$ & $0(0)$ & $<0.001$ \\
\hline Blue-green (type II) & $10(50)$ & $15(75)$ & $2(10)$ & $2(10)$ & \\
\hline Green (type III) & $5(25)$ & $2(10)$ & $11(55)$ & $11(55)$ & \\
\hline Green-yellow-red (type IV) & $0(0)$ & $0(0)$ & $7(35)$ & $7(35)$ & \\
\hline
\end{tabular}

The result are expressed as number (\%); LHBT - long head of the biceps tendon

Table III. Comparison of the mean values of shear wave elastography between LHBT tendinosis and control groups.

\begin{tabular}{llll}
\hline & LHB tendinosis & Pvalues \\
\cline { 2 - 3 } & Transverse & Longitudinal & $<0.001$ \\
\hline & $38.32 \pm 7(28.4-55.9)$ & $39.42 \pm 7.4(28-54)$ & \\
& Control group & $20.62 \pm 4.6(13.3-30)$ & \\
\hline
\end{tabular}

Categorical data are presented as mean \pm standard deviation and range. LHBT - long head of the biceps tendon; SWE - shear wave elastography. 


\section{Discussions}

Tendinosis describes a chronic tendon injury with degeneration at the cellular level and no inflammation [19-22]. Type I collagen fibers are predominantly present in the healthy tendon. Type I collagen is the most important component in tissue elasticity. With tendinosis, type I collagen fibers are thinner and loosely organized [19]. These structural changes are related to tissue damage and include both degenerative areas as well as reactive areas where progressive healing or fibrosis appears $[19,20]$. These degenerative areas on the tendon are seen as an increase in the tendon signal in MRI associated with loss of normal fibrillary pattern [6]. Degenerative changes are seen as hypo- or hyperechoic dishomogeneous thickening, diffuse or focal, of the tendon in B-mode US $[23,24]$. In recent years, many studies have reported connections between the histologic features and tissue elasticity $[25,26]$. Tendinosis is expected to decline in tendon elasticity due to chronic degeneration and fibrosis $[19,20,22]$. SE and SWE provide information on the hardness or softness of the tissue in relation to the state of the disease $[27,28]$.

Seo et al [18] performed SE elastography evaluation of LHBT tendinitis and tendinosis in comparison with B-mode US and found SE to be a potentially clinically useful values in excellent agreement with the conventional US in the differentiation of intratendinous and peritendinous changes. SE and US findings were compared, with transverse and longitudinal ultrasound images were found positive correlatesd. In our study, both SE and SWE in LHBT tendinopathies were evaluated in comparison with high diagnostic value MRI, which is the gold standard diagnostic method in LHBT pathology. However, to our knowledge, both qualitative and quantitative sonoelastographic examination of the LHBT has not been reported in the literature.

In the musculoskeletal system, similar sonoelastography studies of other tendon pathologies have been performed. De Zordo et al [27,29] found sonoelastography to be sensitive in detecting tendon changes in lateral epicondylitis and Achilles tendinopathy compared with US findings. Ooi et al [30] showed an excellent correlation with US findings in Achilles tendinopathy. Klauser et al [31] found a consistent histological comparison between US findings and SE values of common extensor tendinopathy. Park et al [32] reported as a powerful diagnostic tool providing additional information on lateral elbow tendinopathy US findings. Galletti et al [23] reported SE as a complementary method to the US in a study involving extensive tendon pathologies ofthe upper and lower limbs tendons. Seo et al [33] examined in the supraspina- tus tendon for intratendinous and peritendinous changes and established an excellent correlation between MRI and conventional US findings. Lee et al [6] used MRI to evaluate tendinosis grade and compared this with SE, showing that sonoelastography may be a useful diagnostic tool in supraspinatus tendinopathy.

As mentioned, many sonoelastographic studies on tendon pathologies have been performed, mostly compared with US, and most of them used the strain elastography technique, but studies with the SWE technique are more limited. Zhang et al [34] reported a thickening and hardening of patellar tendinopathy on SWE examination of 33 male athletes with patellar tendinopathy compared to healthy volunteers. Petrescu et al [35] evaluated normal and pathologic Achilles tendon by SWE and examined post-operative Achilles tendon with US and MRI.

Our study was a comparison with MRI and both SE and SWE techniques were used. Aubry et al [36] investigated the differences in viscoelastic properties between normal and pathologic Achilles tendons using real-time SWE. Tendon softening assessed using SWE appeared to be highly specific, but sensitivity was relatively low. The tendon pathology described in this study is tendinitis. Tendon pathology in our study is tendinosis.

According to our study, SE and SWE sonoelastography in the diagnosis of LHBT tendinosis was close to the diagnosis from MRI, with a very high sensitivity and specificity and high diagnostic accuracy.

Limitations of the study: first, overrated the results of the study because of the small population; second, the vast majority of patients with biceps tendon pathology are associated with rotator cuff pathologies, and pathological changes in the biceps tendon were related with this chronic process; third, inability to predict the effects of the fluid in the tendon sheath on the results of the sonoelastography; fourth, the images obtained by SE depend on operator-applied pressure, and although the operator's dependence is a known difficulty, we tried to obtain appropriate images by applying some pressure on the probe by monitoring the quality factor; fifth, in MRI, the fluid in the tendon sheath may cause false positive results. Nevertheless, we think that this study is meaningful. Additional comprehensive studies are required with clinical examinations, MRI, arthroscopy and histologic findings, in a comparison with elastography.

No significant difference was found in the elastographic examinations of the fluid and non-fluid portions of the tendon sheath. However, this can be regarded as a limitation of our work as mentioned above. In our study, SE and SWE examinations showed stiffness in the tendons with dishomogeneity. As a matter of fact, there are publications in the literature which show that the dishomogeneity is caused by degeneration and fibrosis $[23,24]$. 
When compared with MRI, sonoelastography showed high sensitivity and specificity in the diagnosis of tendinosis. In LHBT tendinosis, SE and SWE indicated that the resulting LHBT hardness was significantly increased compared to healthy individuals. Accordingly, these measurements can be indicative of significant hardening in LHBT tendinosis. Our study represents the first sonoelastographic study in the literature on LHBT tendinosis that has used both SE and SWE techniques.

In conclusion tendinosis of the LHBT can be diagnosed with conventional US. When SE and SWE were added to the evaluation, we found very high sensitivity and specificity values, close to the MRI diagnosis. SE and SWE may be useful diagnostic tools for LHBT tendinosis when considering usability, cost effectiveness and patient preference compared to MRI.

\section{Conflict of interest: none.}

Funding: Kirikkale University Medical Faculty Scientific Research Project has provided funding for prospective sono-elastography examination (Project no. 2017/031).

\section{References}

1. Ditsios K, Agathangelidis F, Boutsiadis A, Karataglis D, Papadopoulos P. Long head of the biceps pathology combined with rotator cuff tears. Adv Orthop 2012;2012:405472.

2. Patton WC, McCluskey GM 3rd. Biceps tendinitis and subluxation. Clin Sports Med 2001;20:505-529.

3. Ophir J, Cespedes I, Ponnekanti H, Yazdi Y, Li X. Elastography: a quantitative method for imaging the elasticity of biological tissues. Ultrason Imaging 1991;13:111=134.

4. Pesavento A, Perrey C, Krueger M, Ermert H. A time-efficient and accurate strain estimation concept for ultrasonic elastography using iterative phase zero estimation. IEEE Trans Ultrason Ferroelectr Freq Control 1999;46:1057-1067.

5. Magee TH, Gaenslen ES, Seitz R, Hinson GA, Wetzel LH. MR imaging of the shoulder after surgery. AJR Am J Roentgenol 1997;168:925-928.

6. Lee SU, Joo SY, Kim SK, Lee SH, Park SR, Jeong C. Realtime sonoelastography in the diagnosis of rotator cuff tendinosis. J Shoulder Elbow Surg 2016;25:723-729.

7. Middleton WD, Payne WT, Teefey SA, Hildebolt CF, Rubin DA, Yamaguchi K. Sonography and MRI of the shoulder: comparison of patient satisfaction. AJR Am J Roentgenol 2004;183:1449-1452.

8. Dwivedi A, Dhagat PK, Singh SN, Singh S, Yadav N. Role of MRI in a Series of Cases with Biceps Injuries of the Shoulder. J Clin Diagn Res 2017;11:TR01-TR05.

9. İnal M, Tan S, Yumusak EM, Şahan MH, Alpua M, Örnek $\mathrm{K}$. Evaluation of the optic nerve using strain and shear wave elastography in patients with multiple sclerosis and healthy subjects. Med Ultrason 2017;19:39-44.
10. Thitaikumar A, Ophir J. Effect of lesion boundary conditions on axial strain elastograms: a parametric study. Ultrasound Med Biol 2007;33:1463-1467.

11. Tan S, Özcan MF, Tezcan F, et al. Real-time elastography for distinguishing angiomyolipoma from renal cell carcinoma: preliminary observations. AJR Am J Roentgenol 2013;200:W369-W375.

12. Detorakis ET, Drakonaki EE, Tsilimbaris MK, Pallikaris IG, Giarmenitis S. Real-time ultrasound elastographic imaging of ocular and periocular tissues: a feasibility study. Ophthalmic Surg Lasers Imaging 2010;41:135-141.

13. Teber MA, Tan S, Dönmez U, et al. The use of real-time elastography in the assessment of gallbladder polyps: preliminary observations. Med Ultrason 2014;16:304-308.

14. Armstrong CA, Oldham JA. A comparison of dominant and non-dominant hand strengths. J Hand Surg Br 1999;24:421425 .

15. Ok N, Agladioglu K, Gungor HR, et al. Relationship of side dominance and ultrasonographic measurements of pronator quadratus muscle along with handgrip and pinch strength. Med Ultrason 2016;18:170-176.

16. Abe T, Loenneke JP. Handgrip strength dominance is associated with difference in forearm muscle size. J Phys Ther Sci 2015;27:2147-2149.

17. Backhaus M, Burmester GR, Gerber T, et al. Working Group for Musculoskeletal Ultrasound in the EULAR Standing Committee on International Clinical Studies including Therapeutic Trials. Guidelines for musculoskeletal ultrasound in rheumatology. Ann Rheum Dis 2001;60:641-649.

18. Seo JB, Yoo JS, Ryu JW. Sonoelastography findings of biceps tendinitis and tendinosis. J Ultrasound 2014;17:271277.

19. Scott A, Backman LJ, Speed C. Tendinosis: Update on Pathophysiology. J Orthop Sports Phys Ther 2015;45:833841.

20. Bass E. Tendinopathy: why the difference between tendinitis and tendinosis matters. Int $\mathrm{J}$ Ther Massage Bodywork 2012;5:14-17.

21. Churgay CA. Diagnosis and treatment of biceps tendinitis and tendinosis. Am Fam Physician 2009;80:470-476.

22. Kaeding C, Best TM. Tendinosis: pathophysiology and nonoperative treatment. Sports Health 2009;1:284-292.

23. Galletti S, Oliva F, Masiero S, et al. Sonoelastography in the diagnosis of tendinopathies: an added value. Muscles Ligaments Tendons J 2016;5:325-330.

24. Grassi W, Filippucci E, Farina A, Cervini C. Sonographic imaging of tendons. Arthritis Rheum 2000;43:969-976.

25. Çebi Olgun D, Korkmazer B, Kılıç F, et al. Use of shear wave elastography to differentiate benign and malignant breast lesions. Diagn Interv Radiol 2014;20:239-244.

26. Vural M, Acar D, Toprak U, et al. The evaluation of the retrobulbar orbital fat tissue and optic nerve with strain ratio elastography. Med Ultrason 2015;17:45-48.

27. De Zordo T, Lill SR, Fink C, et al. Real-time sonoelastography of lateral epicondylitis: comparison of findings between patients and healthy volunteers. AJR Am J Roentgenol 2009;193:180-185. 
28. Guo X, Liu Y, Li W. Diagnostic accuracy of shear wave elastography for prediction of breast malignancy in patients with pathological nipple discharge. BMJ Open 2016;6: e008848.

29. De Zordo T, Chhem R, Smekal V, et al. Real-time sonoelastography: findings in patients with symptomatic achilles tendons and comparison to healthy volunteers. Ultraschall Med 2010;31:394-400.

30. Ooi CC, Schneider ME, Malliaras P, Chadwick M, Connell DA. Diagnostic performance of axial-strain sonoelastography in confirming clinically diagnosed Achilles tendinosis: comparison with B-mode ultrasound and color Doppler imaging. Ultrasound Med Biol 2015;41:15-25.

31. Klauser AS, Pamminger M, Halpern EJ, et al. Extensor tendinosis of the elbow assessed with sonoelastography: histologic correlation. Eur Radiol 2017;27:3460-3466.

32. Park G, Kwon D, Park J. Diagnostic confidence of sonoelastography as adjunct to greyscale ultrasonography in lateral elbow tendinosis. Chin Med J 2014;127:31103115.

33. Seo JB, Yoo JS, Ryu JW. Sonoelastography findings of supraspinatus tendon in rotator cuff tendinosis without tear: comparison with magnetic resonance images and conventional ultrasonography. J Ultrasound 2015;18:143-149.

34. Zhang ZJ, Ng GY, Lee WC, Fu SN. Changes in morphological and elastic properties of patellar tendon in athletes with unilateral patellar tendinopathy and their relationships with pain and functional disability. PLoS One 2014;9:e108337.

35. Petrescu PH, Izvernariu DA, Iancu C, et al. Evaluation of normal and pathological Achilles tendon by real-time shear wave elastography. Rom J Morphol Embryol 2016;57:785790.

36. Aubry S, Nueffer JP, Tanter M, Becce F, Vidal C, Michel F. Viscoelasticity in Achilles tendonopathy: quantitative assessment by using real-time shear-wave elastography. Radiology 2015;274:821-829. 